\title{
Parasystolic ventricular tachycardia Observations on differential stimulus threshold as possible mechanism for exit block
}

\author{
J. Roelandt and L. Schamroth \\ From the Department of Cardiology, University Hospital, Leiden, and the Department of \\ Cardiology, University Hospital 'Dijkzigt', Rotterdam, The Netherlands; the Baragwanath \\ Hospital and the University of the Witwatersrand, fohannesburg, South Africa
}

Two cases of parasystolic ventricular tachycardia are described. Exit block was demonstrated in one patient manifesting with the temporary disappearance of the ectopic rhythm. This only occurred during faster supraventricular rates induced either by exercise or atrial pacing. These observations reveal a differential stimulus threshold state as a possible mechanism for exit block: a relatively weak stimulus of an ectopic ventricular focus, unlike the stronger channelled supraventricular impulse, is unable to activate the surrounding myocardium during a high threshold phase of the cardiac cycle.

In addition, $a$ 'family resemblance' of $Q R S$ complexes with similar morphology is evident in one of our cases and the published cases of parasystolic ventricular tachycardia associated with normal beats. $A$ similar $Q R S$ morphology has also been reported with right ventricular extrasystoles in normal subjects.

The heart has many pacemaking cells which are situated in the sinoatrial node, the atria, the atrioventricular node, and the ventricles. Only one such pacemaking cell is, however, in control of the heart. This is the pacemaker with the fastest inherent discharge rate. Its impulses reach the slower subsidiary pacemakers before they have the opportunity to 'fire' and abolish their immature impulses prematurely. This ensures that there is only one pacemaker in control of the heart. At times, however, a subsidiary and slower ectopic pacemaking cell develops a protective mechanism which is situated in the immediate vicinity of the ectopic pacemaking cell and is operative throughout its entire cycle. This enables the slower ectopic pacemaker to coexist with the more rapid supraventricular pacemaker - a condition known as parasystole. Though the sinus impulse cannot penetrate into the ectopic pacemaking focus, the ectopic impulse can leave the ectopic focus and activate the surrounding myocardium, when it is responsive consequent to activation by the supraventricular impulses. The ectopic impulses, therefore, manifest intermittently when the myocardium is responsive. And, since the discharge is

Received 12 October 1970. regular, the longer interectopic intervals will be in simple multiples of the shorter interectopic intervals ${ }^{1}$ or the manifest (or calculated) ectopic cycle length. ${ }^{1}$

The independent character of the ectopic rhythm is further evident from the presence of varying coupling intervals: the intervals between the manifest ectopic beats and their preceding sinus beats. This variation reveals that there is no relation between the ectopic and sinus beats. Fortuitous simultaneous invasion of the ventricular myocardium by the sinus and ectopic ventricular impulses will result in ventricular fusion complexes.

Occasionally a parasystolic ventricular impulse may not become manifest though calculation clearly reveals that the ectopic discharge occurs during the non-refractory state of the ventricular myocardium. This is known as exit block: a block in the conduction of the ectopic impulse from the ectopic focus to the surrounding ventricular myocardium.

A parasystolic ventricular discharge may occasionally be rapid. The ectopic ventricular

\footnotetext{
1 An interectopic interval is the interval between two ectopic beats containing intervening sinus beats. The ectopic cycle length is the interval between two consecutive ectopic beats, i.e. with no intervening sinus beats (Scherf and Schott, 1953).
} 
rate may even exceed the sinus rate. It then usurps control of the heart, since the sinus pacemaker enjoys no protection from the faster ectopic impulses. The ectopic rhythm then manifests as an ectopic ventricular tachycardia. When this occurs, the parasystolic character of the rhythm is only evident when the manifest ectopic rhythm intermits. It will then be found that the long interectopic intervals between the periods of manifest ectopic rhythm are in multiples of the ectopic cycle length, thereby indicating that the parasystolic rhythm continues during the apparent intermission, but is not manifest due to the presence of exit block. The arrhythmia is rare.

We report 2 cases of rapid ventricular parasystole, one of which showed a differential stimulus threshold state, thereby revealing a possible mechanism for exit block.

\section{Case reports}

Case I The electrocardiogram (Fig. I - a continuous strip of standard lead II) was recorded from a 22-year-old healthy athletic man during a routine physical examination. There was no history of chest pain or other symptoms. Physical examination was completely normal. The 12-lead conventional electrocardiogram (not illustrated) and the vectorcardiogram of the normally conducted sinus beats (Fig. 2) were completely normal. An exercise test was also normal. Chest $x$-ray showed no abnormalities.

The electrocardiogram (Fig. I) shows normal sinus rhythm complicated by an ectopic ventricu-
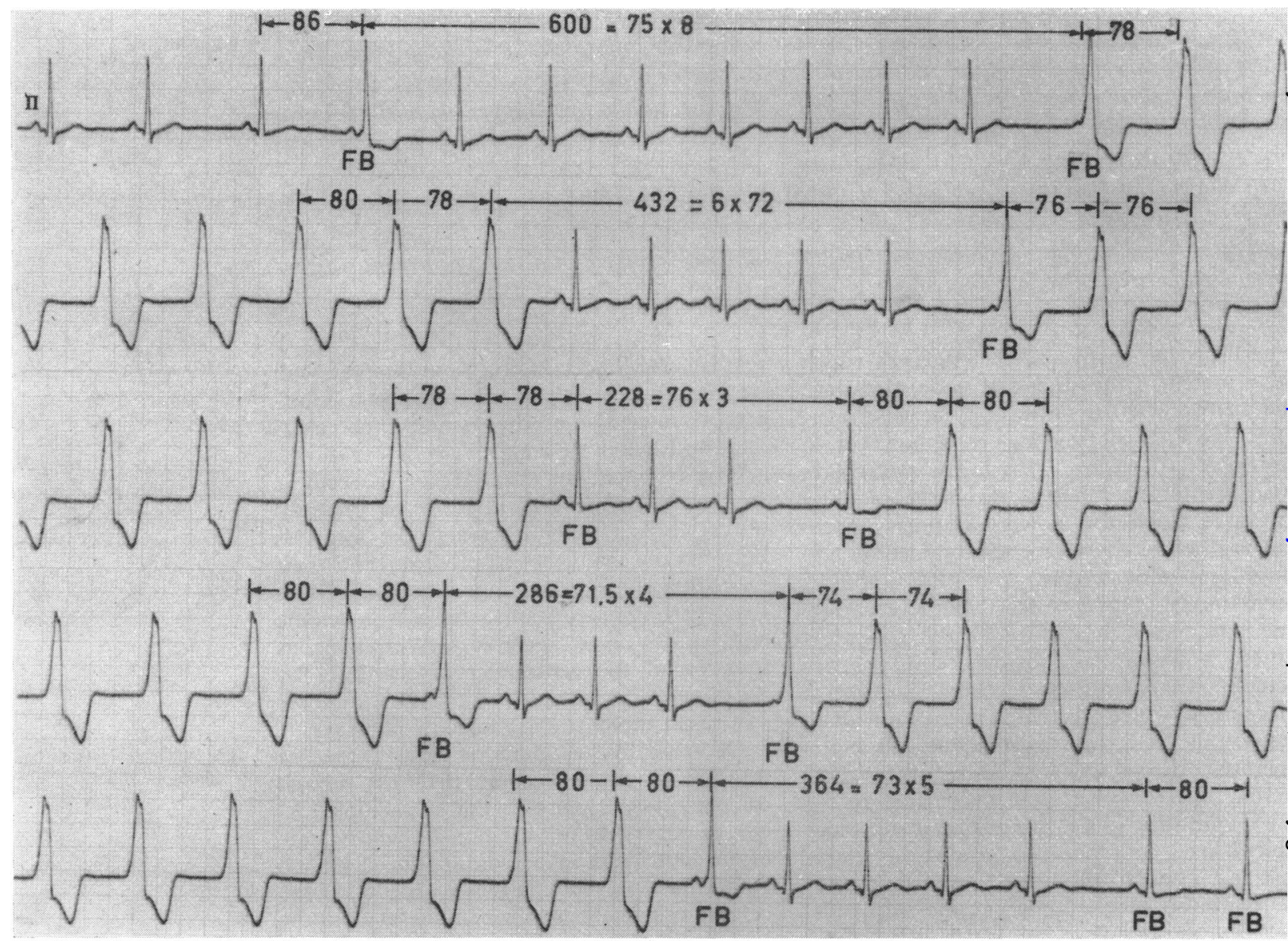

FIG. I (Case I) Electrocardiogram (a continuous recording of standard lead II) showing parasystolic ventricular tachycardia. The normal sinus rhythm varies from 75 to Ioo beats a minute. The ectopic ventricular rhythm is represented by the bizarre $Q R S$ complexes which are unrelated to $P$ waves. The ectopic cycle lengths range from 74 to 80 hundredths of a second. The longer interectopic intervals are in simple multiples of 73.5 (range: $7 I \cdot 5$ to 76$)$ hundredths of a second. Frequent fusion beats $(F B)$ are present. These features reflect the presence of an independent ventricular parasystolic rhythm. 


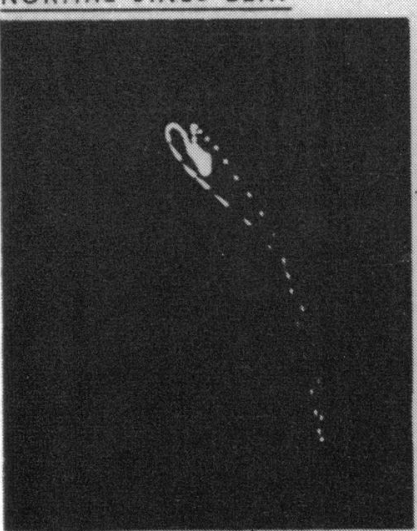

FRONT

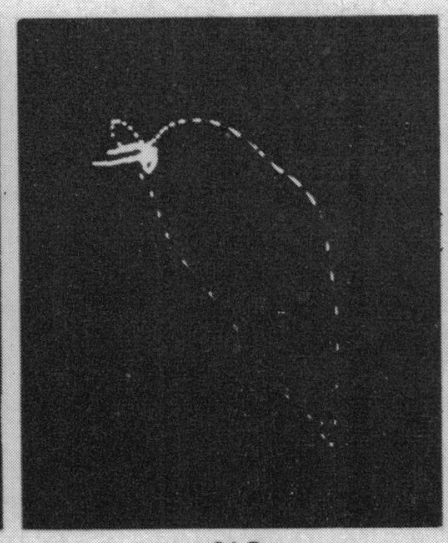

SAC

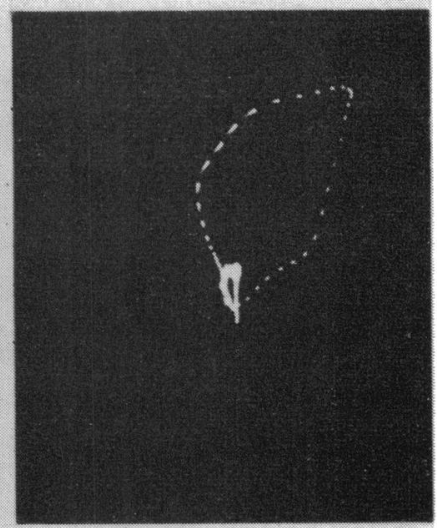

HOR

\section{PARASYSTOLIC BEAT}

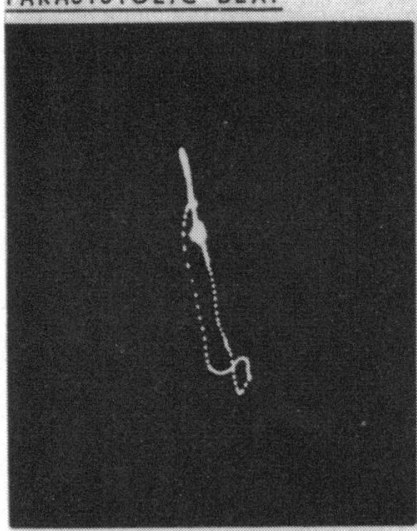

FRONT

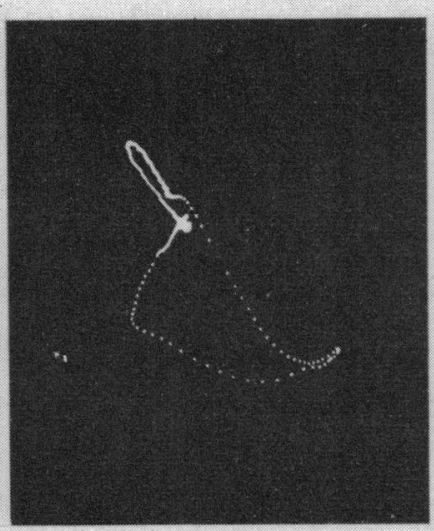

SAC

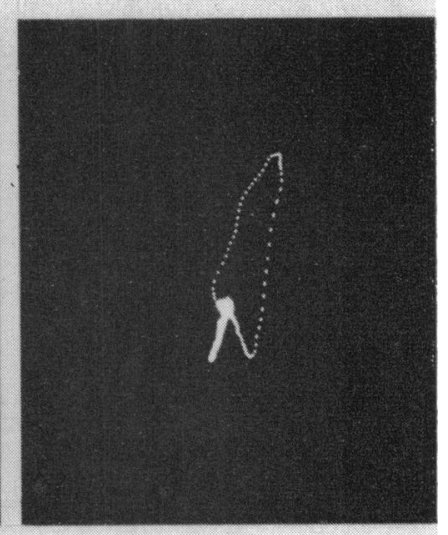

HOR

FIG. 2 (Case I) Vectorcardiograms of the normal sinus and parasystolic beats.

Inscription of the QRS loop of the normal sinus beat in the frontal (FRONT), sagittal (SAG), and horizontal (HOR) plane is normal. The QRS loop of the parasystolic beat shows an atypical left bundle-branch block form. The initial part of the QRS loop is directed inferiorly and anteriorly and is very slowly inscribed. Unlike the vectorcardiogram of typical left bundle-branch block, the horizontal vector runs counterclockwise.

lar rhythm. The normal sinus rhythm is represented, for example, by the first two $P: Q R S$ complexes. The RR intervals of the sinus rhythm range from $60^{1}$ to 80 , reflecting a rate of 75 to 100 beats a minute. The ectopic ventricular rhythm is represented by the bizarre QRS complexes which are unrelated to $P$ waves. The $R R$ intervals of this ectopic ventricular rhythm range from 74 to 80 (mean 78), reflecting a rate of 75 to $8 \mathrm{I}$ beats a minute. The longer interectopic intervals between the periods of manifest ectopic ventricular rhythm are in simple multiples of 73.5 (range $71 \cdot 5$ to 76 ). Frequent fusion beats (FB) are present. These features reflect the presence of an independent ectopic ventricular parasystolic rhythm.

${ }^{1}$ All time intervals are expressed in hundredths of a second, i.e. $60=60$ hundredths of a second.
In effect, the manifest ectopic rhythm reflects an enhanced ventricular rhythm or a ventricular tachycardia, since the normal unenhanced idioventricular rhythm (protected or unprotected) is usually about 30 beats a minute.

The vectorcardiogram of the parasystolic ectopic beats (Fig. 2) shows a QRS loop with an atypical left bundle-branch block form. The initial QRS inscription is very slowly inscribed and directed inferiorly and anteriorly. The main QRS forces are directed inferiorly. Unlike the vectorcardiogram of typical left bundle-branch block, the horizontal vector is inscribed counterclockwise. The $T$ wave loop is in a discordant position. The right ventricular origin of the ectopic beats was also proved phonocardiographically by the reversed splitting of the second heart sound (not illustrated). 


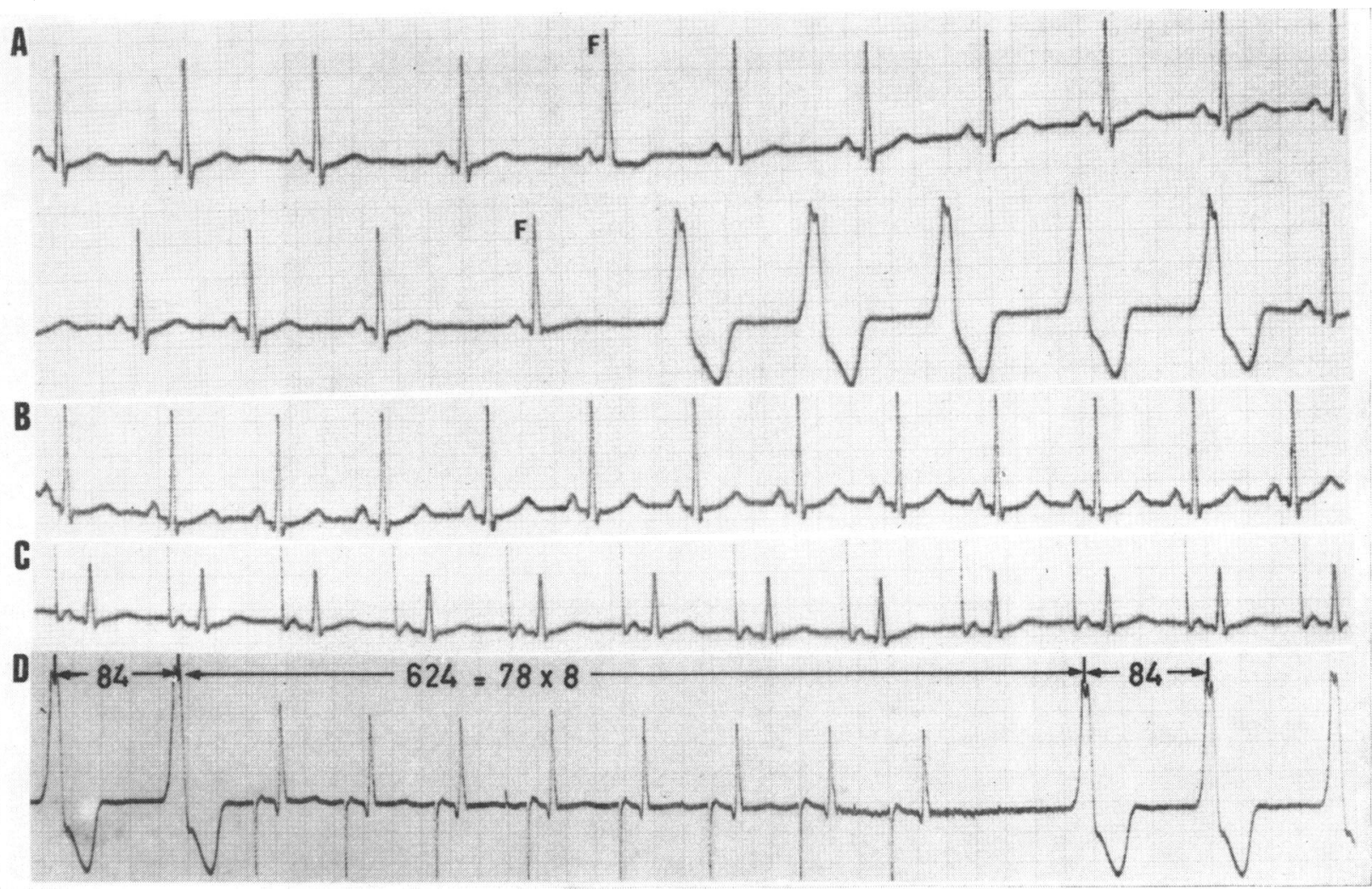

FIG. 3 (Case 2) Electrocardiograms illustrating the influence of different supraventricular rhythms on the advent of exit block. $(A) A$ dominant sinus rhythm at a relatively slow rate. The ectopic rhythm manifests with occasional fusion beats $(F) ;(B)$ sinus tachycardia, induced by exercise; no ectopic activity is manifest; $(C)$ the rhythm recorded during atrial pacing at a rate of 87 beats a minute, no ectopic activity is evident; $(D)$ the rhythm recorded during pacing for a few seconds at a rate of 95 beats a minute. The manifest ectopic rhythm is temporarily abolished. The long interectopic interval is, however, a multiple of the ectopic cycle length, indicating the continuous discharge of the ectopic focus during the apparent intermission.

The aforementioned features were observed during a follow-up period of 3 years.

\section{Effect of exercise}

The patient was exercised and sinus tachycardia induced. No ectopic activity was evident both during the exercise and during a relatively long period of sinus tachycardia which ensued (representative tracing is shown in strip B of Fig. 3). The ectopic rhythm invariably became manifest again when the sinus rhythm slowed.

\section{Effect of atrial pacing}

Under local anaesthesia a $5 \mathrm{~F}$ bipolar electrode catheter was introduced percutaneously into an antecubital vein and fluoroscopically positioned against the lateral wall of the right atrium. The right atrium was then paced by means of a battery-powered variable pacemaker at rates ranging from 70 up to 160 a minute with incre- ments of about Io beats a minute. Pacing was performed for periods of a few seconds (strip D of Fig. 3 shows a pacing rate of 95 beats a minute) to many minutes (strip C of Fig. 3 shows a representative tracing at a pacing rate of 87 beats a minute). The ectopic activity was always suppressed during the short pacing periods and returned always during the sinus pause after the pace run. The long interectopic interval resulting from the temporary disappearance of the ectopic rhythm was always a multiple of the ectopic cycle length (strip D of Fig. 3). There was also no evidence of ectopic ventricular activity during very long periods of atrial pacing.

The temporary disappearance of the ectopic rhythm during fast supraventricular rhythms thus reflects the presence of exit block.

Case 2 The electrocardiogram was recorded from a 50-year-old man during an attack of acute anterolateral myocardial infarction. The tracings 


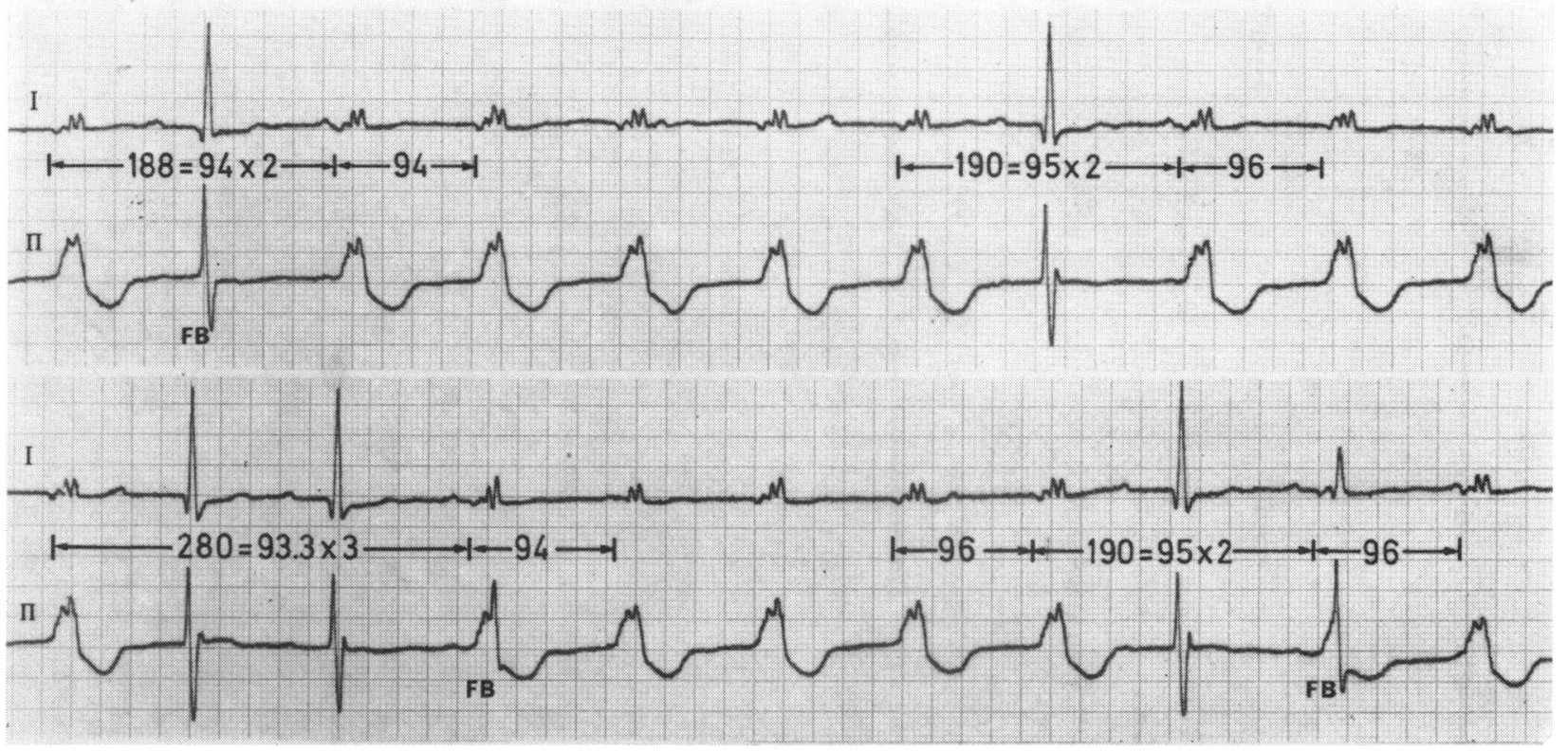

FIG. 4 (Case 2) Electrocardiogram (a continuous tracing of standard leads $I$ and II) showing parasystolic ventricular tachycardia. Sinus bradycardia is present at a rate of 50 beats a minute. The ectopic rhythm, represented by the bizarre $Q R S$ complexes, is independent and dissociated from the sinus rhythm. Capture beats, both complete and incomplete (ventricular fusion beats $-F B$ ), are present. The ectopic cycle length varies from 93.3 to 96 hundredths of a second. Two consecutive capture beats are present in the lower strip. The first, because of its greater prematurity is conducted with a longer PR interval $(0.52 \mathrm{sec})$ than the second $(0.32 \mathrm{sec})$.

(Fig. 4 - continuous strips of standard leads I and II) show an ectopic ventricular rhythm as reflected by the bizarre QRS complexes. These bizarre QRS complexes are dissociated from the $P$ waves of the sinus rhythm. The PP intervals of the sinus rhythm measure 120 , representing a sinus bradycardia with a rate of 50 beats a minute. The RR intervals of the ectopic rhythm measure 94, reflecting a rate of 64 beats a minute. Occasional conducted sinus beats (capture beats) are present.

Some of these capture beats are incomplete, resulting in ventricular fusion complexes (FB). The long interectopic interval between the two consecutive parasystolic beats in the lower strip measures 280 . This is equivalent to three times 93.3: the duration of the ectopic cycle length. When two consecutive capture beats occur (lower strip), the first, due to its greater prematurity, is associated with a longer PR interval (52) than the second (32).

\section{Discussion}

Most cases of ventricular parasystole manifest with a slow ventricular rate, a rate similar to the inherent, unenhanced idioventricular rhythm. In other words, the inherent idioventricular rhythm merely acquires the property of protection, and is thus able to manifest as a parasystolic rhythm. Manifest or calculated ventricular parasystolic rates greater than 60 beats a minute reflect an enhancement of the protected idioventricular rhythm, constituting a parasystolic ventricular tachycardia.

Few clinical examples of manifest or calculated ventricular parasystolic tachycardia have been reported (Katz and Pick, 1956, their Fig. I96 and 214; Scherf and Bornemann, 196I; Schamroth, I962; Chung, Walsh, and Massie, I965; Chung, 1968; Schwartz, 1969). Parasystolic ventricular tachycardia has also been elicited experimentally (Scherf and Chick, I95I; Scherf et al., I95I; Mueller and Baron, I953; Scherf, Blumenfeld, and Yildiz, I962).

Electrocardiographic manifestations Rapid ventricular parasystole may manifest as a ventricular tachycardia. This occurs when the ectopic rate exceeds the sinus rate and each ectopic impulse is able to activate the heart. In other words, the ectopic rhythm is not complicated by exit block and is associated 

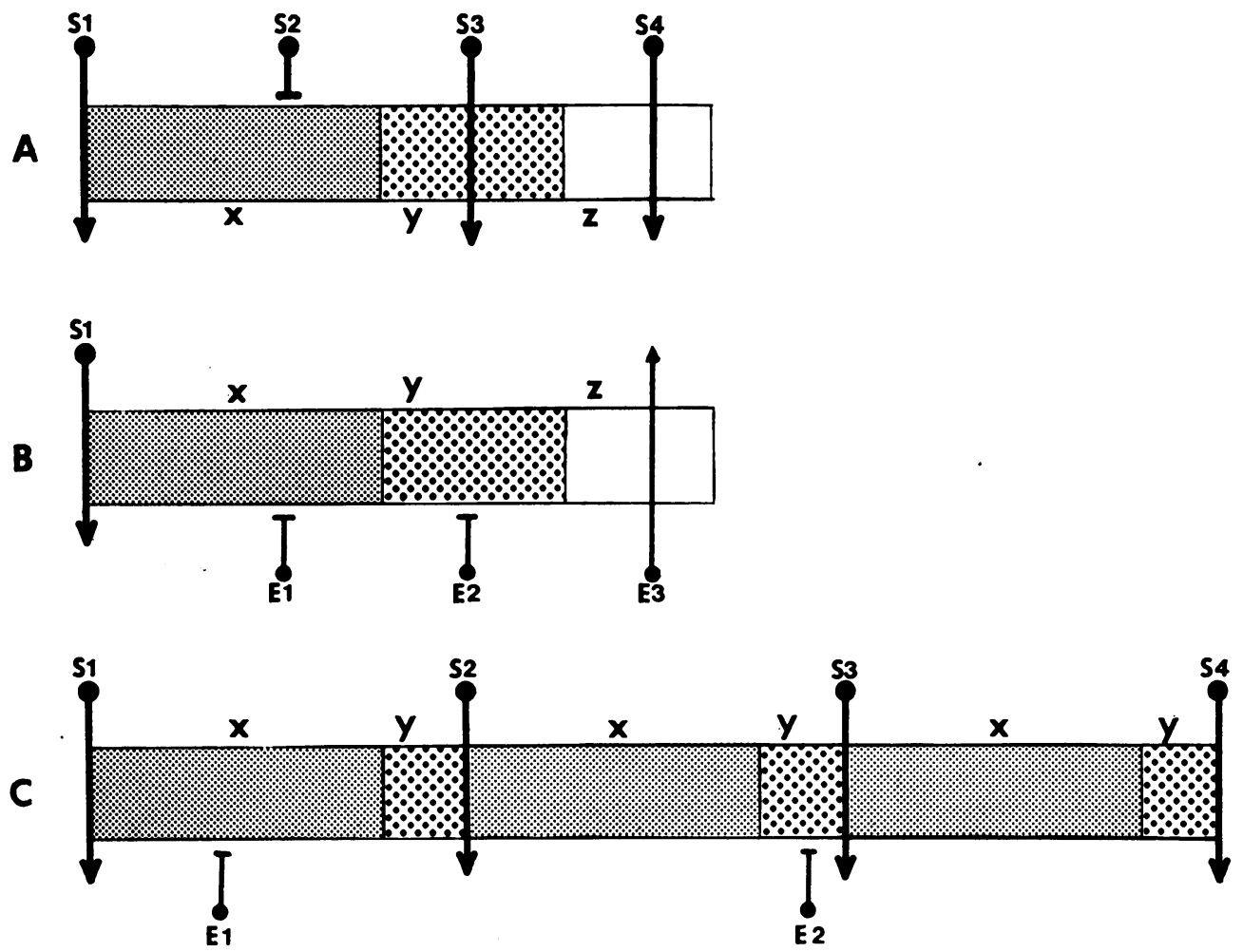

FIG. 5 Diagrams illustrating the principle of differential stimulus-threshold as a possible mechanism for exit block. Diagram A illustrates the effect of a relatively strong supraventricular impulse $(S)$ during the state of absolute refractoriness $(X)$, the state of high threshold refractoriness $(Y)$, and the state of low threshold responsiveness $(Z)$. The impulse is only blocked during the state of absolute refractoriness (impulse $S_{2}$ ). Diagram B illustrates the effect of the relatively weak ectopic impulses $(E)$ during the same periods. The impulse is blocked during both the states of absolute refractoriness $(X)$ and the state of high threshold responsiveness $(Y)$ (impulses $E_{I}$ and $E_{2}$ ). Diagram $C$ illustrates the events during a faster supraventricular rhythm. The sinus cycles shorten and occur during successive zones of high threshold responsiveness $(Y)$. All are, however, conducted to the ventricles since they constitute relatively strong impulses (impulses $S_{1}$ to $S_{4}$ ). When this process is continuous, as occurs during exercise or atrial tachycardia induced by pacing, a zone of low threshold responsiveness cannot occur, and the relatively weak ectopic impulses ( $E_{1}$ and $E_{2}$ ) can therefore not leave their focus. This constitutes exit block.

with I: I exit conduction. The ectopic rhythm will then usurp control of the heart. The parasystolic character of the ventricular tachycardia only becomes evident when the manifest ectopic rhythm intermits. When this occurs, the long interectopic intervals between the periods of manifest tachycardia are in simple multiples of the ectopic cycle length. This indicates that the ectopic rhythm continues during the apparent intermission but is masked by exit block.

Ventricular parasystolic tachycardia may also manifest as a slow parasystolic rhythm. This occurs when the rapid ectopic discharge is masked by exit block. The rapid discharge becomes evident from the following.

(I) Calculation. Calculation and analysis of the long interectopic intervals reveals a short common multiple - a short ectopic cycle length. It must be noted that precisely constant interectopic intervals are rare. It is well known that measurement of the direct parasystolic cycle is usually slightly longer than the calculated ectopic intervals when sinus or other basic beats are present (Chung, 1968). It is our impression that the variation of the interectopic intervals is greater in the cases with apparently normal hearts. 
(2) Dissipation of exit block. The manifest ectopic rate suddenly doubles, thereby revealing the dissipation of a pre-existing $2: 1$ exit block. The manifest or calculated ectopic rate in parasystolic tachycardia is commonly in the range of 60 to roo beats a minute and is, therefore, slower than the usual rate of an extrasystolic ventricular tachycardia. Occasionally, however, the rate of a parasystolic ventricular tachycardia may be as high as 200 beats a minute (e.g. Fig. 194 of Katz and Pick, 1956).

'Family resemblance' The QRS configuration in some cases of rapid ventricular parasystole appears to be the same, giving rise to a 'family resemblance'. This is particularly so in all published cases associated with apparently normal hearts. This 'family resemblance' is present in cases reported by Chung et al. (I965) (their Cases 2 and II), Lamb (1965) (his Fig. 529 to 532), and our Case I. It is interesting to note that Rosenbaum (1969) found right ventricular premature beats with typical and constant electrocardiographic features in normal hearts. The vectorcardiogram of these premature beats showed the same atypical left bundle-branch block form as seen in the ectopic beats of our Case I (Fig. 2). The origin of the ectopic activity seems to be the same, somewhere in the myocardium high in the right ventricle.

A 'family resemblance' has recently been reported in atrial parasystole (Friedberg and Schamroth, 1970).

Mechanism of exit block The circumstantial manifestation of exit block in Case I is of particular significance. The exit block only occurs during periods of sinus tachycardia or during periods of rapid electrically paced supraventricular rhythm. This manifestation is a puzzling feature since, at the very least, synchronous and fortuitous invasion of the ventricles could be expected to occur at times, and occasional ventricular fusion complexes should, therefore, be manifest. If, for example, the ventricles are responsive to supraventricular impulses, they should theoretically be equally responsive to the ectopic ventricular impulses. This is indeed the situation when dominant sinus rhythm occurs with a relatively slow rate. Under these circumstances, occasional ventricular fusion beats are clearly manifest (strip A of Fig. 3). However, no such fusion beats occurred during the rapid supraventricular rhythms (strips B, C, and D of Fig. 3). The absence of manifest ventricular parasystolic rhythm during the relatively rapid supra- ventricular rhythm resulting from sinus tachycardia or atrial pacing may be explained on the basis of a differential stimulusthreshold. The principles are considered below.

Relative stimulus strength of supraventricular and ectopic ventricular impulses The stimulus strength of a conducted supraventricular impulse is greater than that of an ectopic ventricular impulse. This is, in all probability, due to the large cable core of the specialized conducting system - which forms a relatively strong channelling potential. The supraventricular excitation front is thus concentrated, and this results in a strong stimulus 'feed' to the ventricles. The ectopic ventricular impulse originates in the myocardium high in the right ventricle, a region poor in Purkinje fibres. Its excitation front has no such channelling system and is, therefore, relatively weak. This is also evident from the slow and bizarre initial slurring of the ectopic QRS vector loop (Fig. 2).

Differential stimulus threshold After activation, the ventricular myocardium goes through a period of absolute refractoriness which is followed by periods of increasing responsiveness. These responsive periods may be arbitrarily divided into (i) an early - less responsive - period, a period of high threshold responsiveness when the ventricles will only respond to a relatively strong stimulus, and (ii) a later - more responsive - period, a period of relatively low threshold responsiveness when it will also respond to a relatively weak stimulus. The principle is illustrated in Fig. 5. Diagram A illustrates the state of refractoriness and responsiveness following activation of the ventricles by a conducted sinus beat (SI). Ventricular activation is followed by a period of total refractoriness (illustrated by the dark-shaded zone labelled $x$ ) and the ventricles will not respond to any stimulus. This is followed by a period of high threshold responsiveness (illustrated by the light-shaded zone labelled $y$ ), when the ventricles will respond to a relatively strong stimulus. This, in turn, is followed by a period of low threshold responsiveness (illustrated by the unshaded zone labelled $z$ ), when the ventricles will respond to a relatively weak stimulus.

Exit block mechanism If a potential sinus impulse occurs during the period of absolute refractoriness (zone $x$ ), it will be blocked (as illustrated by impulse S2). If a potential sinus impulse occurs during the zone of high threshold responsiveness (zone 
$y)$, it will be conducted, since it constitutes a relatively strong stimulus (as illustrated by impulse $\mathrm{S}_{3}$ ). Similarly, a potential sinus impulse occurring during the zone of low threshold responsiveness (zone $z$ ) will also be conducted (as illustrated by impulse $\mathrm{S}_{4}$ ).

Diagram B (Fig. 5) illustrates the effect of relatively weak potential ectopic ventricular impulses when they occur after a conducted sinus impulse $\left(\mathrm{SI}_{\mathrm{I}}\right.$ ). If a potential ectopic ventricular impulse occurs during the zone of absolute refractoriness (zone $x$ ), it will be blocked (as illustrated by impulse EI). If a potential ectopic ventricular impulse occurs during the zone of high threshold responsiveness (zone $y$ ) it will also be blocked, since it constitutes a relatively weak stimulus (as illustrated by impulse E2). If, however, an ectopic ventricular impulse occurs during the zone of low threshold responsiveness (zone $z$ ), it will be conducted (as illustrated by impulse E3).

Diagram C (Fig. 5) illustrates the effect of fast supraventricular rhythm. Fast supraventricular rhythm results in an abbreviated sinus cycle. Successive sinus impulses (impulses $\mathrm{SI}_{\mathrm{I}}$ to $\mathrm{S}_{4}$ ) thus fall within successive zones of high threshold responsiveness (zone $y)$. And since the conducted supraventricular impulses constitute relatively strong or high threshold stimuli, they are able to activate the ventricles. When this process is continuous as occurs with tachycardia - there is no opportunity for the development of a zone of low threshold responsiveness. Consequently, the ventricles cannot respond to the low threshold stimuli of ectopic ventricular impulses, and exit block occurs.

Exit block is thus due to a weak stimulus relative to a high threshold surrounding myocardium. This mechanism was suggested by
Faltitschek and Scherf (1932), who postulated that exit block depended upon the intensity of the automatic ectopic impulse and the 'irritability' of the tissues surrounding the centre.

\section{References}

Chung, E. K. Y. (1968). Parasystole. Progress in Cardiovascular Diseases, 11, 64.

Chung, K.-Y., Walsh, T. J., and Massie, E. (1965). Ventricular parasystolic tachycardia. British Heart Fournal, 27, 392.

Faltitschek, F., and Scherf, D. (1932). Klinischer Beitrag zur Parasystoliefrage. Wiener Archiv für innere Medizin und deren Grenzgebiete, 23, 269.

Friedberg, H. D., and Schamroth, L. (1970). Atrial parasystole. British Heart fournal, 32, 172.

Katz, L. N., and Pick, A. (1956). Clinical Electrocardiography. Part I. The Arrhythmias. Lea and Febiger, Philadelphia.

Lamb, L. E. (1965). Electrocardiography and Vectorcardiography. W. B. Saunders, Philadelphia and London.

Mueller, P., and Baron, B. (1953). Clinical studies on parasystole. American Heart fournal, 45, 441.

Rosenbaum, M. B. (1969). Classification of ventricular extrasystoles according to form. Fournal of Electrocardiology, 2, 289.

Schamroth, L. (1962). Ventricular parasystole with slow manifest ectopic discharge. British Heart fournal, 24, 731 .

Scherf, D., Blumenfeld, S., and Yildiz, H. (1962). Extrasystoles and parasystole. American Heart fournal, 64, 357.

Scherf, D., and Bornemann, C. (196I). Parasystole with a rapid ventricular center. American Heart fournal, $62,320$.

Scherf, D., and Chick, F. B. (195I). Experimental parasystole. American Heart fournal, 42, 212.

Scherf, D., Chick, F. B., Scharf, M. M., and Terranova, R. (I95I). Further studies on experimental parasystole and extrasystoles in groups. Proceedings of the Society for Experimental Biology and Medicine, 77, 28.

Scherf, D., and Schott, A. (1953). Extrasystoles and Allied Arrhythmias. William Heinemann, London.

Schwartz, E. L. (1969). Ventricular parasystolic tachycardia. Diseases of the Chest, 55, 77. 